\title{
1 Expansion of CRISPR Targeting Sites in Bombyx mori
}

2

3 Baosheng Zeng ${ }^{\mathrm{a}, \mathrm{b}}$, Shuai Zhan ${ }^{\mathrm{a}}$, Yueqiang Wang ${ }^{\mathrm{a}}$, Yuping Huang ${ }^{\mathrm{a}}$, Jun Xu ${ }^{\mathrm{a}, \mathrm{b}}$, Qun Liu ${ }^{\mathrm{a}}$, Zhiqian

$4 \quad \mathrm{Li}^{\mathrm{a}}$, Yongping Huang ${ }^{\mathrm{a}, *}$ and Anjiang Tan ${ }^{\mathrm{a}, *}$

5

$6{ }^{\mathrm{a}}$ Key Laboratory of Insect Developmental and Evolutionary Biology, Institute of Plant Physiology and

7 Ecology, Shanghai Institutes for Biological Sciences, Chinese Academy of Sciences, Shanghai 200032,

8 China

$9{ }^{\mathrm{b}}$ University of Chinese Academy of Sciences, Beijing 100049, China

10 * Correspondence to: Yongping Huang; E-mail: yphuang@ sibs.ac.cn; Anjiang Tan; E-mail:

11 ajtan01@sibs.ac.cn

12

13

14

15

16

17

18

19

20

21

22

23

24

25

26

27

28

29

30

31

32

33

34

35

36

37

(C) 2016. This manuscript version is made available under the Elsevier user license http://www.elsevier.com/open-access/userlicense/1.0/ 


\section{Abstract}

The CRISPR/Cas9 system has been proven as a revolutionary genome engineering tool. In most cases, single guide RNA (sgRNA) targeting sites have been designed as GN19NGG or GGN18NGG, because of restriction of the initiation nucleotide for RNA Pol III promoters. Here, we demonstrate that the U6 promoter from a lepidopteran model insect, Bombyx mori, effectively expressed the sgRNA initiated with any nucleotide bases (adenine, thymine, guanine or cytosine), which further expands the CRISPR targeting space. A detailed expansion index in the genome was analysed when N20NGG was set as the CRISPR targeting site instead of GN19NGG, and revealed a significant increase of suitable targets, with the highest increase occurring on the $\mathrm{Z}$ sex chromosome. Transfection of different types of N20NGG sgRNAs targeting the enhanced green fluorescent protein (EGFP) combined with Cas9, significantly reduced EGFP expression in the $\mathrm{BmN}$ cells. An endogenous gene, BmBLOS2, was also disrupted by using various types of N20NGG sgRNAs, and the cleavage efficiency of N20NGG sgRNAs with different initial nucleotides and GC contents was evaluated in vitro. Furthermore, transgenic silkworms expressing Cas9 and sgRNAs targeting the BmBLOS2 gene were generated with many types of mutagenesis. The typical transparent skin phenotype in knock-out silkworms was stable and inheritable, suggesting that N20NGG sgRNAs function sufficiently in vivo. Our findings represent a renewal of CRISPR/Cas9 target design and will greatly facilitate insect functional genetics research.

Keywords: CRISPR/Cas9, U6 promoter, sgRNA, transgene, Bombyx mori

8

(1)

60

61

2

63

64

65

66
67 


\section{Introduction}

The newly developed type II prokaryotic CRISPR/Cas9 system has been used for genome engineering in both model and non-model organisms (Cong et al., 2013; Feng et al., 2013; Friedland et al., 2013; Gratz et al., 2013; Hwang et al., 2013; Mali et al., 2013b; Wang et al., 2013a). The mechanism of single-guide RNA (sgRNA) guiding the Cas9 nuclease to cleave specific sites in the genome is well understood (Hsu et al., 2014; Sander and Joung, 2014). Cas9 targeting requires a 20-nucleotide sgRNA sequence that is complementary to the target and a protospacer-adjacent motif (PAM). Theoretically, any N20-PAM sequence in the genome could be set as a potential target. The expression of sgRNAs with a desired sequence is an essential step for CRISPR technology, and the RNA polymerase type III promoter U6 is commonly used for this purpose. In mammals, the U6 promoter drives the expression of sgRNAs with the requirement of a guanine nucleotide for transcription initiation, thus constraining the sgRNA target site to GN19NGG. Ran et al. provided an alternative way by adding one $\mathrm{G}$ at +1 , thus making GN20NGG the target sequence can be efficiently utilized in mammals (Ran et al., 2013c). However, there are no further reports to study the potential side effects of the added 5'G, which artificially induces one mismatch, or whether it could be extended to other classes. Other commonly used promoters for short RNAs in vitro transcription, such as SP6, T7 and T3, also require one or two starting guanine nucleotide(s) (Adhya et al., 1981; Ma et al., 2014a; Melton et al., 1984; Pleiss et al., 1998). Recently, utilizing the ribozyme self-processing capacity, the RNA polymerase II promoter was used to express sgRNAs through a ribozyme-gRNA-ribozyme(RGR) gene system to produce mature sgRNAs (Gao and Zhao, 2014). Additionally, the H1 pol III promoter is an alternative option for sgRNA transcription, which could initiate both guanine and adenine (Ranganathan et al., 2014). In Drosophila, the Pol II nanos promoter has been used to express sgRNAs by adding the 5' and 3' untranslated region (UTR) of nanos gene to the upstream and downstream of sgRNA sequence (Ren et al., 2013).

The domestic silkworm Bombyx mori is a lepidopteran model insect for genetic research (Tan et al., 2013). In recent years, numerous genetic manipulation techniques have been established in B. mori, including transposon-based transgenesis, RNAi, zinc finger nucleases (ZFNs) and transcription activator-like effector nucleases (TALENs) (Sajwan et al., 2013; Takasu et al., 2010; Tamura et al., 2000). The CRISPR/Cas9 system has also been established in B. mori, both in cell lines and in vivo (Liu et al., 2014; Ma et al., 2014b; Wang et al., 2013b). The in vitro transcription of sgRNAs by the T7 
promoter require the target sequence of GGN19NGG, and only GN19NGG sgRNAs expressed with the U6 promoter were applied, which substantially limits the extended usage of the CRISPR/Cas9 system in short coding genes or non-coding RNAs loss of function researches (Ho et al., 2014; Zhao et al., 2014).

In the present study, we demonstrate that the ability of B. mori U6 promoter (Tanaka et al., 2009) to express sgRNAs beginning with the other three nucleotides ( $T, A$ and $C$ ). The potential expansion of CRISPR sites in the B. mori genome was calculated and analysed. The specifically designed N20NGG sgRNAs under the control of the U6 promoter cooperated with the ubiquitously expressed Cas9 protein effectively knocked out both exogenous and endogenous genes in vitro and in vivo. The transparent skin phenotype induced through disruption of BmBLOS2 by N20NGG type sgRNAs (combined with Cas9) was observed and inheritable. Finally, the cleavage efficiency of sgRNAs with different initial nucleotides with different GC contents was also evaluated to provide possible principles for future sgRNAs target design.

\section{Materials and methods}

\subsection{Bioinformatics analysis}

To determine the potential sgRNA target sites in the B. mori genome, the occurrence of AN19NGG, TN19NGG, GN19NGG, CN19NGG and N20NGG in each chromosome was calculated and plotted with Circos (v0.52, http://circos.ca/). Then, we evaluated the mean and median distance values of two adjacent GN19NGG and N20NGG sites. The frequencies of the GN19NGG and N20NGG sites in B. mori genes were also analysed and compared. We also examined the density of GN19NGG and N20NGG in all identified B. mori mRNA and microRNAs. Finally, the genomes of several other representative insect species, including the fruit fly, beetle, honeybee and pea aphid, were also similarly evaluated. The genome and annotation of the silkworm were downloaded from SilkDB. The genomes of the additional insects were obtained from FlyBase (for Drosophila melanogaster), BeetleBase (for Tribolium castaneum), HGD (for Apis mellifera) and AphidBase (for Acyrthosiphon pisum). We used custom Perl scripts to search all of the potential CRISPR sites in the genome and calculated the frequency and distance. The CRISPR sites at genes or miRNAs were determined by BEDtools (intersectBed, v2.17.0). 


\subsection{Silkworms and embryo injections}

The B. mori strain Nistari, which is multivoltine and nondiapausing, was used in all of the experiments. The larvae were reared with fresh mulberry leaves or an artificial diet under standard conditions (Tan et al., 2013). The Cas9 and sgRNA expressing vectors were extracted using a QIAGEN Plasmid Midi Kit (QIAGEN, Hilden, Germany) and purified by phenol chloroform. The mixture of Cas9-sgRNA plasmids and helper plasmids (plasmids which express the piggyBac transposase, final concentration of each vector was $300 \mathrm{ng} / \mu \mathrm{l}$ ) was microinjected into preblastoderm $\mathrm{G} 0$ embryos within 8 hours after oviposition and microinjection was performed according to our previous report (Ling et al., 2014; Tan et al., 2013). For each independent group, 640 preblastoderm embryos were injected, then the injected embryos were incubated at $25{ }^{\circ} \mathrm{C}$ in a humidified chamber for 10 to 11 days until larval hatching (Tan et al., 2013).

\subsection{Plasmid construction}

To generate plasmids for the single-strand annealing (SSA) assay, we constructed seven pGL (firefly luciferase) plasmids within two 870-bp repeat sequences combined with different artificial sgRNA targets. The CMV promoter in the original pGL plasmid (Promega, Madison, WI) was replaced by IE1 promoter(Li et al., 2015a). Then the pGL plasmids containing 870 bp repeat sequence were constructed by two steps of cloning: 1) insert the pGL-part-1(including the first section of GL and first 870 bp repeat sequence) between NheI and BglII; 2) ligate the second part (sgRNA target, the other 870 bp repeat sequence and the rest part of GL ORF sequence) with BglII and BamHI (or XbaI). All the primers used were listed in Table s4.The U6::sgRNA scaffold::pol III terminator sequence was obtained using the U6 promoter as a template (U6 promoter was synthesized based on the sequence reported by Tanaka et al.)(Tanaka et al., 2009) and was amplified with forward primer located at the U6 promoter and reverse primer (sgRNA targeting sequence included) (Table s4), then sub-cloned into the pJET Blunt vector and verified by sequencing. The Cas9 ORF sequence was cloned from the PTD1-Cas9 plasmid(ViewSolid Biotech, Beijing, China) and sub-cloned into pBac[IE1-DsRed2-IE1-SV40](Li et al., 2015a) to achieve pBac[IE1-DsRed2 -IE1-Cas9-SV40] with AatII and ApaI. The vectors used for transgenic transformation were generated by inserting a U6-sgRNA expression cassette into pBac[IE1-DsRed2-IE1-Cas9-SV40] with KpnI and obtained pBac[IE1-DsRed2-U6-sgRNA-IE1-Cas9 -SV40]. The pBac[IE1-EGFP-SV40] was constructed based on pBac[IE1-DsRed2-SV40] by using 


\subsection{BmN cell culture and transfection}

A stable BmN cell line was maintained at $27^{\circ} \mathrm{C}$ with the $\mathrm{TC} 100$ insect cell medium (PAN-Biotech, Aidenbach, Germany) supplemented with $10 \%$ foetal bovine serum (FBS) (Thermo Fisher Scientific, Waltham, MA). The plasmids (including plasmids used for in vitro knock out and SSA assay experiments) used for the transfection in the cells were extracted and purified with phenol chloroform. Transfection was performed using an Effectene Transfection Reagent kit following the manufacturer's recommended instructions (QIAGEN, Hilden, Germany). In total, 400 ng of DNA were transfected per well of a 24-well plate. Twenty-four hours post-transfection, the medium containing transfection reagents was replaced with the new growth medium. Cells were assessed by fluorescence microscopy or harvested for genomic DNA extraction 72 hours after transfection.

\subsection{SSA dual luciferase reporter assay}

For each well of a 24-well plate, $100 \mathrm{ng}$ of pGL reporter plasmid (containing the structure of 870 bp repeat sequence, sgRNA targets and 870 bp repeat sequence), $100 \mathrm{ng}$ of pRL control plasmid(Promega, Madison, WI), 100 ng of Cas9 expression plasmid(pBac[IE1-dsRed-IE1-Cas9-SV40 ]) and 100 ng of sgRNA vector (U6-sgRNA expression plasmids) were transfected (Cradick et al., 2014). Seventy-two hours after transfection, cells were washed with phosphate-buffered saline (PBS) and collected with lysis buffer after vigorous shaking for $15 \mathrm{~min}$. The latter procedure was performed according to the manufacturer's protocol (Dual-Luciferase ${ }^{\circledR}$ Reporter Assay kit, Promega, Madison, WI). The mean of the relative luciferase expression (Firefly luciferase (GL)/Renilla luciferase (RL)) of the control was set as 1 .

\subsection{Surveyor analysis (T7 endonuclease I assay)}

Polymerase chain reaction (PCR) was performed to amplify the fragments surrounding the sgRNA targeting sites from the different groups of genomic DNA. A hybridization reaction was performed with a $19 \mu \mathrm{l}$ mixture containing $200 \mathrm{ng}$ of the PCR product, $2 \mu \mathrm{l}$ of NEbuffer $2(50 \mathrm{mM} \mathrm{NaCl}, 10 \mathrm{mM}$ Tris- $\mathrm{HCl}, 10 \mathrm{mM} \mathrm{MgCl} 2$ and $1 \mathrm{Mm}$ DTT; NEB, Ipswich, MA) and $\mathrm{ddH}_{2} 0$ in a PCR cycler using the 
following programme: $95^{\circ} \mathrm{C}$ for $5 \mathrm{~min}$, ramp down to $85^{\circ} \mathrm{C}$ at $-2^{\circ} \mathrm{C} / \mathrm{s}$, ramp down to $25^{\circ} \mathrm{C}$ at $-0.1^{\circ} \mathrm{C} / \mathrm{s}$, and hold at $4{ }^{\circ} \mathrm{C}$. Then, the mixture was treated with $1 \mu \mathrm{l}$ of T7 endonuclease I (NEB) at $37{ }^{\circ} \mathrm{C}$ for 15 min, and the reaction was terminated by adding $2 \mu \mathrm{l}$ of $0.25 \mathrm{M}$ EDTA. Subsequently, the reaction mixture was loaded for electrophoresis on a 1.5\% agarose gel and was analysed under UV light. The intensity of the cleavage bands and the non-cutting band were measured by using the gel analysis software Quantity One (Bio-Rad, Hercules, California)

\subsection{Molecular characterization of target loci mutations}

Another portion of the PCR products prepared for theT7 endonuclease I assay was ligated into the pJET-Blunt vector and sequenced to validate the detailed genome engineering event, such as a deletion or an insertion. To investigate the mutants in vivo, the larvae that exhibited a mosaic transparent skin phenotype were selected, the genomic DNA of the epidermis was extracted after incubated with proteinase K (Thermo Fisher Scientific, Waltham, MA) and purified by SDS lysis-phenol extraction, then treated with RNaseA (Thermo Fisher Scientific, Waltham, MA)(Ling et al., 2014). DNA fragments around the BmBLOS2 sgRNA target sites were amplified with KOD-Plus polymerase (TOYOBO, Japan) and sequenced.

\subsection{Inverse PCR}

As the piggyBac transposon randomly induces insertion at TTAA sites in the genome, inverse PCR was performed to localize the exact insertion site of each transgenic silkworm line. As the inserted regions of the piggyBac vectors do not have a BfuCI site, $4 \mu \mathrm{g}$ of genomic DNA of the transgenic lines was digested with $\mathrm{BfuCI}\left(\mathrm{NEB}\right.$, Ipswich, MA) at $37^{\circ} \mathrm{C}$ and recovered. The digested genome was ligated with T4 ligase (Thermo Fisher Scientific, Waltham, MA) at $16{ }^{\circ} \mathrm{C}$ overnight. Two primers of opposite directions were designed at the piggyBac 5'-end arm and were used to amplify the self-ligated digested genomic fragment (plasmid sequence and genomic sequence).The PCR products were subsequently sequenced to determine the insertion sites of the piggyBac transposon.

\section{Results}

\subsection{Analysis of CRISPR targeting sites in the genome}

To determine the potential expansion of sgRNA targeting sites, we performed a bioinformatics 
analysis to evaluate the increase of CRISPR targeting sites in the silkworm genome when using N20NGG instead of GN19NGG. Previously, Chen et al. reported that $82.66 \%$ of the genes and $79.87 \%$ of the promoter sequences of B. mori contain N20NGG targets (Chen et al., 2014). We found that the TN19NGG site resulted in the highest percentage $(\sim 30.4 \%)$ of selectable CRISPR sites in the genome (excluding the mitochondrial genome), whereas the CN19NGG resulted in the lowest percentage $(\sim 17.7 \%)$ (Fig. 1A, Fig. s1, Table s1). Of all the chromosomes analysed, chromosome 23 exhibited the highest frequency of CRISPR sites, whereas chromosome 2 presented the lowest. Notably, the most significant expansion of the CRISPR targeting sites was occurred in the $\mathrm{Z}$ sex chromosome, which exhibited a 3.75-fold increase (Fig. 1A, Fig. s1, Table s1).

We also calculated the average genome-wide targeting densities and compared the mean distances of two adjacent CRISPR sites for GN19NGG (59 bp), AN19NGG (58 bp), TN19NGG (57 bp), CN19NGG (82 bp) and N20NGG (14 bp) (Figs. 1B and C). We found a 2.54-fold increase in the CRISPR sites in all of the coding genes (Fig. 1C). In addition, a 4.58-fold increase was noted for the examined 206 microRNA genes and we found 36 genes could be targeted with AN19NGG, TN19NGG or CN19NGG but not GN19NGG (Fig. 1C). Of all the 206 microRNA genes, 202 of them could be targeted with N20NGG sgRNAs and only four microRNA genes could not find any CRISPR targeting sites. The expansion of CRISPR sites in the B. mori genome is useful for identifying more suitable sites for genome editing, and particularly enables a loss of function analysis for small RNAs.

In addition, we performed a similar analysis in another four insect species in which the U6 promoter is commonly used (fruit fly, beetle, honeybee and pea aphid). Although the genome size is different, all of the four insects exhibited a similar result as that in silkworm (Fig. 1D). The increase of CRISPR targeting sites was demonstrated in all insects, with a 2.95 -fold increase in the fruit fly, a 3.88-fold increase in the beetle, a 3.18-fold increase in the honeybee and a 4.45 -fold increase in the pea aphid (Figs. 1D and E). Consequently, the expansion of available CRISPR sites in the insects' genome facilitates more precise genomic editing and correction of mutated alleles.

\subsection{Specific cleavage of EGFP using sgRNAs with different initial nucleotides}

Formerly, when the U6-expressed sgRNAs were used in the CRISPR/Cas9 system, the chosen target is limited to GN19NGG or GGN18NGG, according to the design rules which applied to the mammalian U6 promoter. As the U6 promoter in $B$. mori is also commonly used to drive the expression 
of short hairpin RNAs (shRNAs), the transcription of designed shRNAs may not be initiated with guanine(Tanaka et al., 2009). Therefore, we examined whether the U6 promoter could express sgRNAs that begin with the other three nucleotides in cells. We designed four different sgRNAs that target the open reading frame (ORF) of EGFP, denoted EGFP-sgRNA-A, T, G, and C (Fig. 2A). Three different plasmids (equimolar ratio of Cas9, sgRNAs and EGFP expression vector) were transfected into BmN cells (Fig. 2A). Compared with the control group (only transfected with Cas9 and EGFP expressing plasmids, Fig. 2B), all of the cells treated with sgRNAs exhibited a significant decrease of EGFP fluorescence signals due to the disruption of the EGFP coding sequence after colony formation (Fig. 2B, Fig. s2).

Although all of the sgRNAs down-regulated the expression of EGFP, the sgRNAs whose transcription from the U6 promoter initiated with different nucleotides exhibited diverse efficiency (Fig. 2B). According to the loss of the EGFP signal strength, the sgRNAs transcription was not severely affected if the transcription initiated with guanine or cytosine. By contrast, the cells with sgRNAs that initiated with adenine or thymine showed a relatively moderate loss of EGFP signals or weak EGFP signals, particularly in the adenine started sgRNA group (Fig. 2B). The Cas9 expression vector containing the dsRed expression cassette exhibited the same dsRed signal intensity in all groups, which demonstrated that the EGFP expression down-regulation by CRISPR/Cas9 was irrelevant in terms of the transfection efficiency (Fig. 2B). These results suggest that the B.mori U6 promoter induced transcription of N20NGG sgRNAs can specifically and effectively disrupt exogenous gene expression

\subsection{Deletion of the endogenous BmBLOS2 gene with N20NGG sgRNAs in vitro}

We next sought to test the possibility of disrupting an endogenous gene with N20NGG sgRNAs. We chose BmBLOS2 (B. mori biogenesis of lysosome-related organelles complex 1, subunit 2), which has been used to validate the CRISPR/Cas9 system as an efficient genetic marker gene in $B$. mori (Wang et al., 2013b). We designed four sgRNAs, BmBLOS2-sgRNA-A, T, G, and C, that targeted exons 1 to 4, respectively (Fig. 3A). To estimate the targeting efficiency, we performed a T7EI assay on the amplified sequences surrounding the target site in the genome. All of the four sgRNAs showed clear cleavage bands with different intensity, indicating the variant cutting efficiency (Fig. 3C). The Sanger sequencing results revealed various types of mutations, such as a small deletion or an insertion. 
The adenine-initiated sgRNA induced mutations with a small deletion of 3, 9 and 13 bp at the targeting site. The thymine-initiated sgRNAs caused two short 3 and $7 \mathrm{bp}$ and one another long 39 bp deletions. BmBLOS2-sgRNA-G and C not only induced several base pairs of small deletions but also insertions, such as two insertions of 68 or $77 \mathrm{bp}$ (Fig. 3D). These data suggested that the U6-driven sgRNAs that are initiated with any of the four nucleotides could cause single site mutations.

In addition to evaluating the possibility of inducing small deletions with N20NGG type sgRNAs, we also detected large fragment deletion (LFD) events. Two types of sgRNAs were combined (e.g., AT, AG, AC, TG, TC and GC) for transfection to cleavage the genome at two sites (Fig. s3 A and B). The sequence gap between two targeting sites ranged from 717 to 6,176 bp (AT: 2,575 bp, AG: 3,315 bp, AC: 6,176 bp, TG: 717 bp, TC: 3,578 bp and GC: 2,838 bp) (Fig. s3D), and the deletion events of each gap were detected in all combination groups (Fig. s3 C and D). Together, these results demonstrate that B. mori endogenous genes can be disrupted (including small deletion, insertion and LFD) by U6-driven sgRNAs that begin with $\mathrm{A}, \mathrm{T}, \mathrm{G}$ or $\mathrm{C}$.

\subsection{Cleavage efficiency of sgRNAs beginning with different nucleotides and containing different GC} contents

To evaluate the cleavage efficiency of the sgRNAs that begin with different nucleotides, we performed an SSA dual luciferase reporter assay that is conventionally used for this purpose. We chose one CRISPR targeting site from $B m B L O S 2$ that was previously validated in our laboratory as a positive control (Wang et al., 2013b). Mimic luciferase targets were constructed by inserting another 870 bp sequence (the repeat of one part of the luciferase sequence) into the luciferase ORF. The artificially designed sgRNA target sites were located in the middle of the two 870 bp repeat sequences (Fig. 4A). We designed seven different sgRNAs sites, four of them begin with A, T, G or C, and two extra targeting sites, which included AT and GC-rich sites with guanine as the first nucleotide. The target with a mutated PAM sequence was treated as a negative control (Fig. 4B). After the artificial target cleavage by Cas9 protein, the DNA repair mechanism restores the reporter luciferase gene, and the level of cleavage and repair can be evaluated by measuring the activity of luciferase.

The luciferase reporter assay results showed that sgRNAs beginning with thymine, guanine or cytosine induced a relatively high cleavage level, whereas sgRNAs beginning with adenine induced a low-level cleavage (Fig. 4C). Moreover, sgRNAs with a high GC content exhibited moderate efficiency, 
which may be due to the requirement of more energy to unwind the three hydrogen bonds between guanine and cytosine in the DNA double helix. However, sgRNAs with high AT ratios exhibited higher cleavage and reached the same level as sgRNAs with T, G or C (Fig. 4C). These results suggest that U6 promoter-driven $\operatorname{sgRNAs}$ that begin with any of the four types of nucleotides induce a double-strand break at specific targeting sites but with variable cutting efficiency.

\subsection{N20NGG sgRNAs efficiently induce sequence specific disruptions in vivo}

We constructed four piggyBac-based vectors that carried Cas9 (with an IE1 promoter) and an N20NGG type sgRNA expression cassette (with the U6 promoter) for obtaining constitutively CRISPR/Cas9 manufacturing transgenic silkworms (Fig. 5A). Previous reports have shown that the loss of BmBLOS2 via ZFNs, TALENs or direct injection of in vitro synthesized Cas9 mRNA and sgRNA induces transparent skin or oily silkworm phenotype (Sajwan et al., 2013; Takasu et al., 2010; Wang et al., 2013b). Using a fluorescence microscope (Nikon AZ100), we screened several larvae by detecting the expression of dsRed2 for each injection group (larvae screened as transgenic individuals for IE1-Cas9-U6-BLoS2-sgRNA-A, T, G and C, respectively: 6 larvae, 5 larvae, 9 larvae and 16 larvae). We observed the transparent skin phenotype in all transgenic Cas9 and N20NGG type sgRNAs expression silkworms in G1. Compared with the wild-type silkworms, transgenic BmBLOS2 knockout individuals exhibited a mosaic pattern of transparent skin over the entire body (Fig. 5B). The G1 larvae carrying the Cas9 gene and sgRNA-coding sequence exhibited a fine mosaic pattern of oily parts evenly distributing over the entire body, while irregular sizes of oily parts were randomly observed in the G0 larvae microinjected with Cas9 mRNA and sgRNA (Wang et al., 2013b). This phenotypic difference might be due to the difference in the delivery of Cas9 proteins and sgRNA. In the transgenic larvae, the expression of the transgene is ubiquitous and continuous but presumably weak, while the disruption of the target gene is achieved for a relatively short period of time in the embryos directly microinjected with the Cas9 mRNA and sgRNA. We observed several types of transparent skin phenotypes in the G2 transgenic silkworms, including the chimeric oily skin (the same as in G1), completely transparent skin and yin-yang oily skin (the skin of the half part body is normal, whereas the other part is transparent) (Fig. s5), suggested that the mutant is inheritable.

The mutations at the targeted region of the genome were measured by analysing the T7EI assay results and the details of the mutation events that were confirmed by Sanger sequencing (Fig. 5C, Fig. 
s6). These results suggest that N20NGG sgRNAs induce expected mutants. The transgene insertion sites of the different lines were investigated using inverse PCR, and the results show that the insertion occurs at either two unidentified genes or two exons of one unidentified gene, which does not contribute to the transparent skin appearance of silkworms (Fig. s7). These results suggest that N20NGG sgRNAs can be applied in future genomic research by using the transgene-based CRISPR/Cas system in B. mori (Li et al., 2015b).

\section{Discussion}

The CRISPR/Cas9 system has been extensively applied in functional gene analysis, gene therapy and genome-scale screening (Hsu et al., 2014). The typical Cas9-sgRNA recognition system requires an exact 20-base pairing and NGG PAM sequences. Alternative Cas9 proteins from other bacteria, such as Neisseria meningitidis and Streptococcus thermophilus, require a longer length of pairing bases and PAM sequences other than NGG (Hou et al., 2013). For sgRNA targeting sites selection, the 5'-end nucleotide composition is critical in target recognition and interaction between sgRNA and the Cas9 protein (Nishimasu et al., 2014). The 5'-end nucleotide also influences the repression efficiency in the CRISPR interference system (Larson et al., 2013; Mali et al., 2013a; Qi et al., 2013; Ran et al., 2013b).

In mammals, the most commonly used U6 promoter only drives the sgRNA initiated with guanine (Ding et al., 2013). Recently, the mammalian H1 promoter was reported to drive sgRNAs beginning with both adenine and guanine, thus doubling the sgRNA targeting sites (Ranganathan et al., 2014). RNA polymerase II promoters also used to obtain mature sgRNAs (Gao and Zhao, 2014), which can be used to achieve precise gene modification in cell- or tissue-specific manners.

Here, we demonstrate that the utility of the B. mori U6 promoter overrode the limitation of the requirement of guanine at the +1 transcription initiation site, which is different with mammalian U6 promoter. Our bioinformatics analysis revealed a significant increase of the potential sgRNA targeting sites for coding genes and non-coding RNAs in the $B$. mori genome. This result will help us to identify more available targeting sites in specific region of interest, including short genes and small RNAs. Our in vitro results revealed that U6-directed sgRNAs initiating with $\mathrm{A}, \mathrm{T}, \mathrm{G}$ or $\mathrm{C}$ induced mutations when targeting both exogenous and endogenous genes, thus providing reliable alternatives for designing CRISPR targets in genetic engineering. Furthermore, in vivo experiments provided direct evidence of 
using non-guanine-initiated sgRNAs combined with Cas9 to disrupt endogenous gene with stable and inheritable phenotypes. Consequently, the B. mori U6 promoter may be leveraged to express more CRISPR sites than previously imagined.

A previous report showed that the mouse U6 promoter can initiate transcription using $\mathrm{G}$, or in some cases A, located at positions -1 to at least +2 (Ma et al., 2014a), but it remains unclear whether the transcription initiation of insects' U6 promoter works in the same pattern. A comparative analysis of the mechanisms of the insect U6 promoter, which exhibited a less strict starting-base bias than the mammalian promoters, is of interest. We hypothesize that the insect U6 promoter is much longer than the mammal, and thus carries more regulatory elements which may help to achieve transcription initiation with any nucleotide. A deletion assay can be applied to identify these elements to further reveal the differences between the insect and mammalian RNA Pol III promoter.

The usage of the Cas9 nickase coupled to two sgRNAs or dCas9-FokI combined with a pair of sgRNAs is a new approach that can be used to reduce off-target effects (Ran et al., 2013a; Tsai et al., 2014). The increase of sgRNA targeting space will facilitate the identification of more appropriate target sites with an optimal spacer sequence. To our knowledge, this is the first evidence that CRISPR targets exclude GN19NGG in silkworm genome engineering. This approach will particularly facilitate gene activation analysis through the CRISPR activation (CRISPRa) system, which requires multiple sgRNAs to target the 5'-UTR region within a limited length of approximately 500 bp (Cheng et al., 2013; Gilbert et al., 2014). The increasing focus on the development of the CRISPR/Cas9 systems stresses the importance of increasing CRISPR targeting sites, including the expansion of the CRISPR targeting library. Our results provide a reliable reference for designing CRISPR targets for genetic engineering in silkworms.

\section{Acknowledgements}

This work was supported by grants from National Basic Research Program of China (2012CB114101), the National Science Foundation of China (31420103918 and 31530072) and the External Cooperation Program of BIC, Chinese Academy of Sciences (Grant No. GJHZ201305).

\section{References}

Adhya, S., Basu, S., Sarkar, P., Maitra, U., 1981. Location, Function, and Nucleotide-Sequence of a 
Promoter for Bacteriophage-T3 Rna-Polymerase. P Natl Acad Sci-Biol 78, 147-151.

Chen, L., Tang, L., Xiang, H., Jin, L., Li, Q., Dong, Y., Wang, W., Zhang, G., 2014. Advances in genome editing technology and its promising application in evolutionary and ecological studies. GigaScience 3, 24.

Cheng, A.W., Wang, H., Yang, H., Shi, L., Katz, Y., Theunissen, T.W., Rangarajan, S., Shivalila, C.S., Dadon, D.B., Jaenisch, R., 2013. Multiplexed activation of endogenous genes by CRISPR-on, an RNA-guided transcriptional activator system. Cell research 23, 1163-1171.

Cong, L., Ran, F.A., Cox, D., Lin, S., Barretto, R., Habib, N., Hsu, P.D., Wu, X., Jiang, W., Marraffini, L.A., Zhang, F., 2013. Multiplex genome engineering using CRISPR/Cas systems. Science 339, 819-823.

Cradick, T.J., Antico, C.J., Bao, G., 2014. High-throughput cellular screening of engineered nuclease activity using the single-strand annealing assay and luciferase reporter. Methods Mol Biol 1114, 339-352.

Ding, Q.R., Regan, S.N., Xia, Y.L., Oostrom, L.A., Cowan, C.A., Musunuru, K., 2013. Enhanced Efficiency of Human Pluripotent Stem Cell Genome Editing through Replacing TALENs with CRISPRs. Cell stem cell 12, 393-394.

Feng, Z.Y., Zhang, B.T., Ding, W.N., Liu, X.D., Yang, D.L., Wei, P.L., Cao, F.Q., Zhu, S.H., Zhang, F., Mao, Y.F., Zhu, J.K., 2013. Efficient genome editing in plants using a CRISPR/Cas system. Cell research 23, 1229-1232.

Friedland, A.E., Tzur, Y.B., Esvelt, K.M., Colaiacovo, M.P., Church, G.M., Calarco, J.A., 2013. Heritable genome editing in C. elegans via a CRISPR-Cas9 system. Nat Methods 10, 741-743.

Gao, Y.B., Zhao, Y.D., 2014. Self- processing of ribozyme- flanked RNAs into guide RNAs in vitro and in vivo for CRISPR- mediated genome editing. Journal of integrative plant biology 56, 343-349.

Gilbert, L.A., Horlbeck, M.A., Adamson, B., Villalta, J.E., Chen, Y., Whitehead, E.H., Guimaraes, C., Panning, B., Ploegh, H.L., Bassik, M.C., Qi, L.S., Kampmann, M., Weissman, J.S., 2014. Genome-Scale CRISPR-Mediated Control of Gene Repression and Activation. Cell 159, 647-661.

Gratz, S.J., Cummings, A.M., Nguyen, J.N., Hamm, D.C., Donohue, L.K., Harrison, M.M., Wildonger, J., O'Connor-Giles, K.M., 2013. Genome Engineering of Drosophila with the CRISPR RNA-Guided Cas9 Nuclease. Genetics 194, 1029-+.

Ho, T.T., Zhou, N., Huang, J., Koirala, P., Xu, M., Fung, R., Wu, F., Mo, Y.Y., 2014. Targeting non-coding RNAs with the CRISPR/Cas9 system in human cell lines. Nucleic acids research.

Hou, Z.G., Zhang, Y., Propson, N.E., Howden, S.E., Chu, L.F., Sontheimer, E.J., Thomson, J.A., 2013. Efficient genome engineering in human pluripotent stem cells using Cas9 from Neisseria meningitidis. Proceedings of the National Academy of Sciences of the United States of America 110, 15644-15649. Hsu, P.D., Lander, E.S., Zhang, F., 2014. Development and applications of CRISPR-Cas9 for genome engineering. Cell 157, 1262-1278.

Hwang, W.Y., Fu, Y.F., Reyon, D., Maeder, M.L., Tsai, S.Q., Sander, J.D., Peterson, R.T., Yeh, J.R.J., Joung, J.K., 2013. Efficient genome editing in zebrafish using a CRISPR-Cas system. Nature biotechnology 31, 227-229.

Larson, M.H., Gilbert, L.A., Wang, X.W., Lim, W.A., Weissman, J.S., Qi, L.S., 2013. CRISPR interference (CRISPRi) for sequence-specific control of gene expression. Nature protocols 8, 2180-2196.

Li, Z., Zeng, B., Ling, L., Xu, J., You, L., Aslam, A.F., Tan, A., Huang, Y., 2015a. Enhancement of Larval RNAi Efficiency by Over-expressing Argonaute2 in Bombyx mori. International journal of 
biological sciences 11, 176-185.

Li, Z.Q., You, L., Zeng, B.S., Ling, L., Xu, J., Chen, X., Zhang, Z.J., Palli, S.R., Huang, Y.P., Tan, A.J., 2015b. Ectopic expression of ecdysone oxidase impairs tissue degeneration in Bombyx mori. P Roy Soc B-Biol Sci 282.

Ling, L., Ge, X., Li, Z., Zeng, B., Xu, J., Aslam, A.F., Song, Q., Shang, P., Huang, Y., Tan, A., 2014. MicroRNA Let-7 regulates molting and metamorphosis in the silkworm, Bombyx mori. Insect biochemistry and molecular biology 53, 13-21.

Liu, Y., Ma, S., Wang, X., Chang, J., Gao, J., Shi, R., Zhang, J., Lu, W., Zhao, P., Xia, Q., 2014. Highly efficient multiplex targeted mutagenesis and genomic structure variation in Bombyx mori cells using CRISPR/Cas9. Insect biochemistry and molecular biology 49, 35-42.

Ma, H.M., Wu, Y.G., Dang, Y., Choi, J.G., Zhang, J.L., Wu, H.Q., 2014a. Pol III Promoters to Express Small RNAs: Delineation of Transcription Initiation. Mol Ther-Nucl Acids 3.

Ma, S., Chang, J., Wang, X., Liu, Y., Zhang, J., Lu, W., Gao, J., Shi, R., Zhao, P., Xia, Q., $2014 b$. CRISPR/Cas9 mediated multiplex genome editing and heritable mutagenesis of BmKu70 in Bombyx mori. Scientific reports $4,4489$.

Mali, P., Aach, J., Stranges, P.B., Esvelt, K.M., Moosburner, M., Kosuri, S., Yang, L.H., Church, G.M., 2013a. CAS9 transcriptional activators for target specificity screening and paired nickases for cooperative genome engineering. Nature biotechnology 31, 833-+.

Mali, P., Yang, L., Esvelt, K.M., Aach, J., Guell, M., DiCarlo, J.E., Norville, J.E., Church, G.M., 2013 b. RNA-guided human genome engineering via Cas9. Science 339, 823-826.

Melton, D.A., Krieg, P.A., Rebagliati, M.R., Maniatis, T., Zinn, K., Green, M.R., 1984. Efficient in vitro synthesis of biologically active RNA and RNA hybridization probes from plasmids containing a bacteriophage SP6 promoter. Nucleic acids research 12, 7035-7056.

Nishimasu, H., Ran, F.A., Hsu, P.D., Konermann, S., Shehata, S.I., Dohmae, N., Ishitani, R., Zhang, F., Nureki, O., 2014. Crystal Structure of Cas9 in Complex with Guide RNA and Target DNA. Cell 156, 935-949.

Pleiss, J.A., Derrick, M.L., Uhlenbeck, O.C., 1998. T7 RNA polymerase produces 5' end heterogeneity during in vitro transcription from certain templates. RNA 4, 1313-1317.

Qi, L.S., Larson, M.H., Gilbert, L.A., Doudna, J.A., Weissman, J.S., Arkin, A.P., Lim, W.A., 2013. Repurposing CRISPR as an RNA-guided platform for sequence-specific control of gene expression. Cell 152, 1173-1183.

Ran, F.A., Hsu, P.D., Lin, C.Y., Gootenberg, J.S., Konermann, S., Trevino, A.E., Scott, D.A., Inoue, A., Matoba, S., Zhang, Y., Zhang, F., 2013a. Double Nicking by RNA-Guided CRISPR Cas9 for Enhanced Genome Editing Specificity. Cell 154, 1380-1389.

Ran, F.A., Hsu, P.D., Lin, C.Y., Gootenberg, J.S., Konermann, S., Trevino, A.E., Scott, D.A., Inoue, A., Matoba, S., Zhang, Y., Zhang, F., 2013b. Double Nicking by RNA-Guided CRISPR Cas9 for Enhanced Genome Editing Specificity (vol 154,pg 1380, 2013). Cell 155, 479-480.

Ran, F.A., Hsu, P.D., Wright, J., Agarwala, V., Scott, D.A., Zhang, F., 2013c. Genome engineering using the CRISPR-Cas9 system. Nature protocols 8, 2281-2308.

Ranganathan, V., Wahlin, K., Maruotti, J., Zack, D.J., 2014. Expansion of the CRISPR-Cas9 genome targeting space through the use of $\mathrm{H} 1$ promoter-expressed guide RNAs. Nature communications 5.

Ren, X.J., Sun, J., Housden, B.E., Hu, Y.H., Roesel, C., Lin, S.L., Liu, L.P., Yang, Z.H., Mao, D.C., Sun, L.Z., Wu, Q.J., Ji, J.Y., Xi, J.Z., Mohr, S.E., Xu, J., Perrimon, N., Ni, J.Q., 2013. Optimized gene editing technology for Drosophila melanogaster using germ line-specific Cas9. Proceedings of the 
National Academy of Sciences of the United States of America 110, 19012-19017.

Sajwan, S., Takasu, Y., Tamura, T., Uchino, K., Sezutsu, H., Zurovec, M., 2013. Efficient disruption of endogenous Bombyx gene by TAL effector nucleases. Insect biochemistry and molecular biology 43, $17-23$.

Sander, J.D., Joung, J.K., 2014. CRIRISPR-Cas systems for editing, regulating and targeting genomes. Nature biotechnology 32, 347-355.

Takasu, Y., Kobayashi, I., Beumer, K., Uchino, K., Sezutsu, H., Sajwan, S., Carroll, D., Tamura, T., Zurovec, M., 2010. Targeted mutagenesis in the silkworm Bombyx mori using zinc finger nuclease mRNA injection. Insect biochemistry and molecular biology 40, 759-765.

Tamura, T., Thilbert, C., Royer, C., Kanda, T., Abraham, E., Kamba, M., Komoto, N., Thomas, J.L., Mauchamp, B., Chavancy, G., Shirk, P., Fraser, M., Prudhomme, J.C., Couble, P., 2000. Germline transformation of the silkworm Bombyx mori L-using a piggyBac transposon-derived vector. Nature biotechnology 18, 81-84.

Tan, A., Fu, G., Jin, L., Guo, Q., Li, Z., Niu, B., Meng, Z., Morrison, N.I., Alphey, L., Huang, Y., 2013. Transgene-based, female-specific lethality system for genetic sexing of the silkworm, Bombyx mori. Proceedings of the National Academy of Sciences of the United States of America 110, 6766-6770.

Tanaka, H., Fujita, K., Sagisaka, A., Tomimoto, K., Imanishi, S., Yamakawa, M., 2009. shRNA expression plasmids generated by a novel method efficiently induce gene-specific knockdown in a silkworm cell line. Molecular biotechnology 41, 173-179.

Tsai, S.Q., Wyvekens, N., Khayter, C., Foden, J.A., Thapar, V., Reyon, D., Goodwin, M.J., Aryee, M.J., Joung, J.K., 2014. Dimeric CRISPR RNA-guided FokI nucleases for highly specific genome editing. Nature biotechnology 32, 569-576.

Wang, H.Y., Yang, H., Shivalila, C.S., Dawlaty, M.M., Cheng, A.W., Zhang, F., Jaenisch, R., $2013 a$. One-Step Generation of Mice Carrying Mutations in Multiple Genes by CRISPR/Cas-Mediated Genome Engineering. Cell 153, 910-918.

Wang, Y., Li, Z., Xu, J., Zeng, B., Ling, L., You, L., Chen, Y., Huang, Y., Tan, A., 2013b. The CRISPR/Cas system mediates efficient genome engineering in Bombyx mori. Cell research 23, 1414-1416.

Zhao, Y., Dai, Z., Liang, Y., Yin, M., Ma, K., He, M., Ouyang, H., Teng, C.B., 2014. Sequence-specific inhibition of microRNA via CRISPR/CRISPRi system. Scientific reports 4, 3943.

. 
(A) Circos plot depicting the frequency of CRISPR targeting sites in the B. mori genome. The outside numbers and the letter circle depict the silkworm chromosome ideograms ( $\mathrm{Z}$ chromosome and autosome: 2-28, without the $\mathrm{W}$ chromosome). The inner yellow circle represents all of the annotated coding genes. GN19NGG (orange), AN19NGG (blue), CN19NGG (purple), and TN19NGG (green) are located further inwards. (B) The density of the GN19NGG and N20NGG sgRNAs in the genome by calculating and comparing the frequency of two adjacent CRISPR targeting sites (GN19NGG and N20NGG). The x-axis shows the frequency number of the possible CRISPR targeting sites when the distance between two adjacent GN19NGG and N20NGG sites (the blue bar stands for GN19NGG and red bar represents for N20NGG, and part of the red bar was overlapped with the blue bar) is in the range of 1-9 bp or the latter series distances in the genome. (C) The median distance of two adjacent CRISPR targeting sites of five different groups of sgRNAs in the silkworm genome, genes and microRNAs. The bar with a different colour corresponds to a certain type of sgRNA shown in the legend. (D) Bar plot shows the frequency of different types of sgRNAs in the genome of five representative insects: silkworm, fruit fly, beetle, honeybee and pea aphid. The bar with a different colour corresponds to a certain type of sgRNA shown in the legend. (E) The median distance of two adjacent CRISPR targeting sites of five different types of sgRNAs in the genome of five representative insects (listed in D). The bar with a different colour corresponds to a certain type of sgRNA shown in

\section{Fig. 2. Disruption of EGFP expression by Cas9 with sgRNAs with different initial nucleotides}

550 Sequences of the four sgRNAs targeting EGFP ORF are shown above (EGFP-sgRNA-A, T, G, and C).

551 The lower section describes plasmids that were transfected into BmN cells. The IE1-Cas9 plasmid also carries a dsRed expression cassette. (B) EGFP fluorescence microscopy suggests successful CRISPR targeting by the U6-sgRNA with different nucleotides at the 5'-end. EGFP fluorescence was severely weakened after the disruption of EGFP by Cas9 with specific sgRNAs, whereas the dsRed fluorescence was not influenced. The exposure time for all of the photos is $180 \mathrm{~ms}$. 
Fig. 3. Site-specific mutation of the BmBLOS2 in BmN cells with N20NGG sgRNAs

558 (A) Four designed N20NGG-type sgRNAs (BmBLOS2-sgRNA-A, T, G and C) are located in exons 1

to 4 , which are indicated by the boxes with different colours. (B) Cas9 was expressed downstream of

560 the robust and ubiquitous IE1 promoter and co-transfected with the sgRNA vector into BmN cells.

561 Cells were harvested after 72 hours for further analysis. (C) Cleavage events and cutting efficiency were evaluated with a T7EI assay. The T7EI assay of BmBLOS2-sgRNA-A and T is presented in the left panels. The first and third rows are the control band, and the second and fourth rows depict the sgRNA-A and T band. The right section presents BmBLOS2-sgRNA-G and C. (D) Mutation events were confirmed by sequencing. Both small deletion and insertion events occurred at specific sites, and at least 3 different types of mutations are listed here.

Fig. 4. Evaluation of the cleavage efficiency of N20NGG sgRNAs with a single strand-annealing (SSA) assay

(A) Flow diagram of the SSA assay. The firefly luciferase protein was separated into two parts (GL-P1 and GL-P2), and an 870-bp repeat sequence was presented in each part. Then, a 23 bp CRISPR target was inserted in the middle. The IE1 promoter-induced Cas9 expressing vector was the same as in Fig. 3 and different types of sgRNAs (in B) were expressed by the U6 promoter. Renilla luciferase was used as the control. (B) Seven groups of different sgRNAs were analysed. Red letters indicate the first nucleotide of the sgRNA, and blue letters denote the PAM sequence. The underlined letters represent the designed AT and GC-rich type sgRNAs. (C) Normalized luciferase activity suggests the cleavage efficiency of various designed sgRNAs in (B). The luciferase activity in the control group was set as 1 .

Fig. 5. Silkworms with oily skin phenotype obtained by transgenic expression of BmBLOS2 sgRNAs targeting N20NGG sequences

(A) Constructs for piggyBac based transgenic transformation. The marker is dsRed with the IE1 promoter and HR5 as the enhancer. The Cas9 protein is also ubiquitously expressed with IE1. U6 drives N20NGG-type BmBLOS2 sgRNAs (U6- BmBLOS2-sgRNAs (A, T, G, and C)) (B) Silkworms of 
586 were photographed with UV light with a RFP filter. (C) Mutation confirmation. Deletion events (at 587 least 3 mutants, marked as M1, M2 and M3; WT stands for the original sequence) of four lines are 588 listed.

589 
A

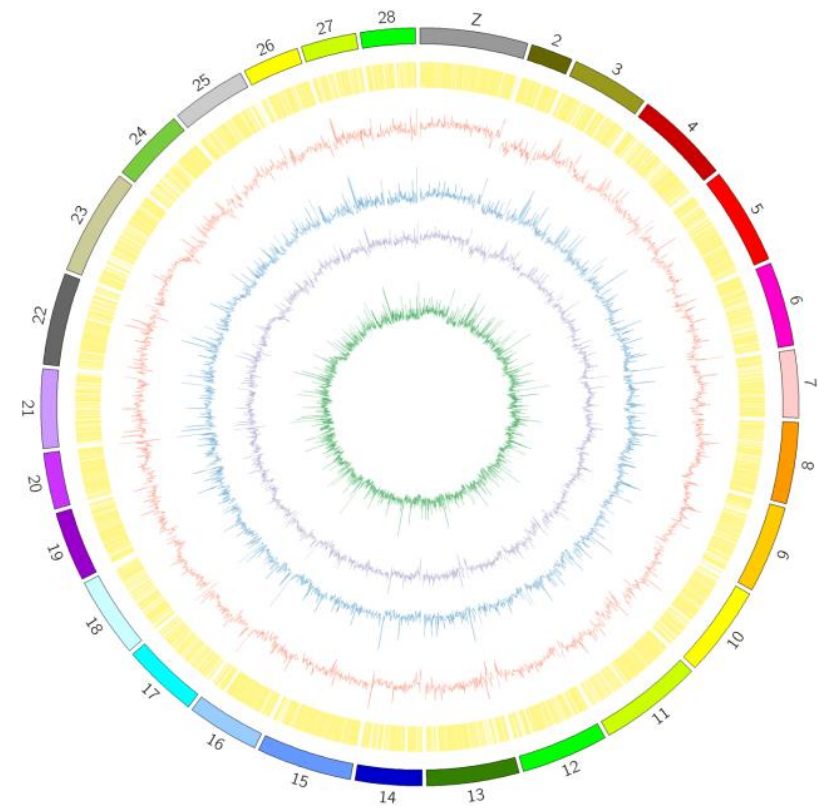

\section{B}

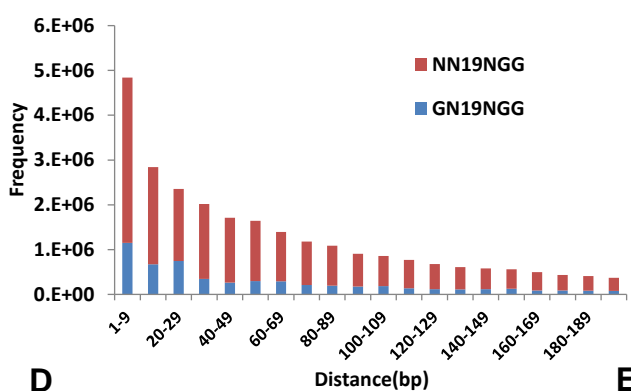

D

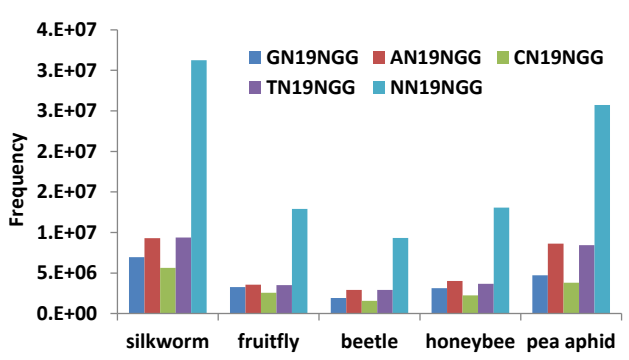

C

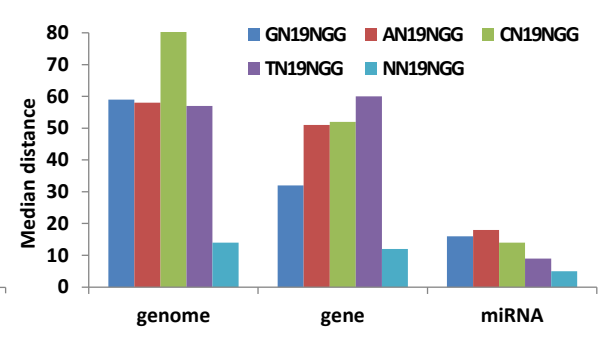

E

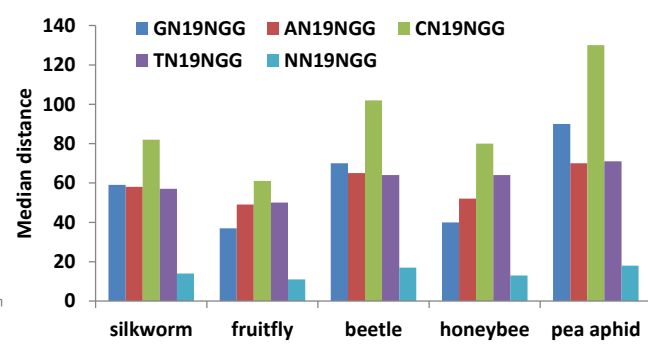

Fig. 1. Genome-wide pattern of CRISPR targeting sites 
EGFP-sgRNA-A : AAGGAGGACGGCAACATCCTGGG EGFP-sgRNA-T: TACCAGCAGAACACCCCCATCGG EGFP-sgRNA-G : GAGCTGGACGGCGACGTAAACGG EGFP-sgRNA-C : CATGCCCGAAGGCTACGTCCAGG
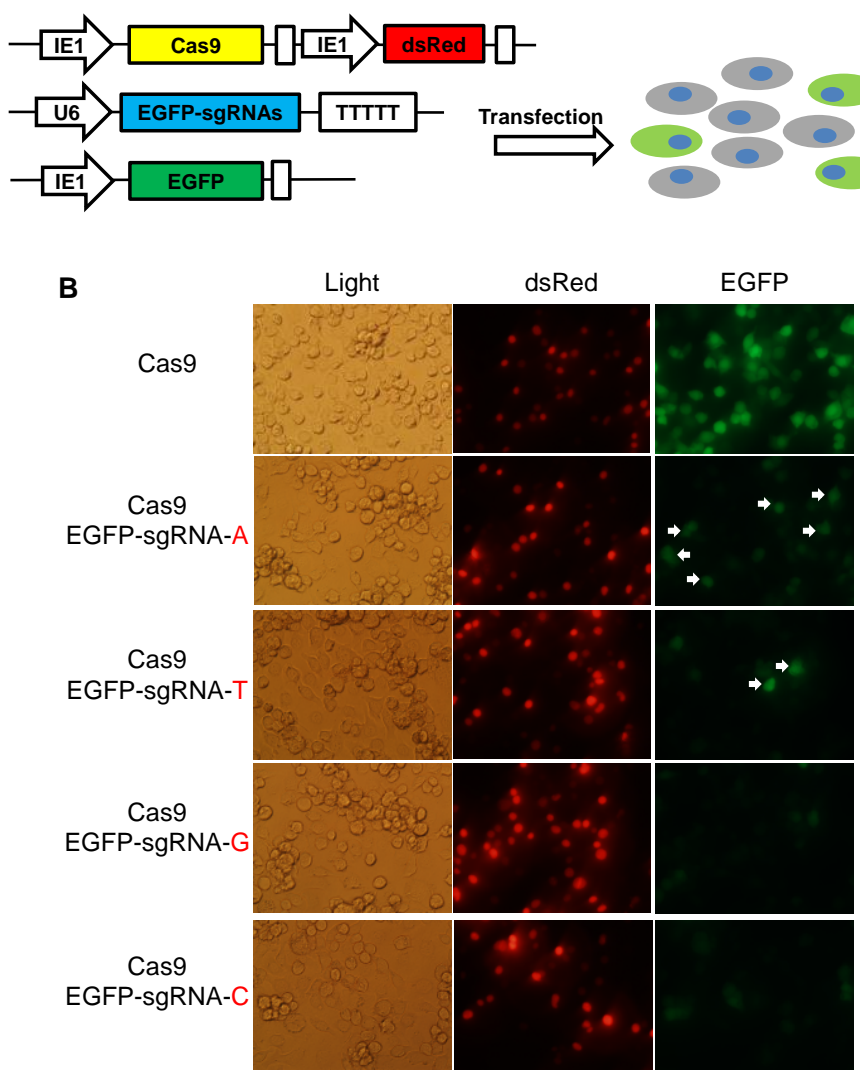

606

Fig. 2. Disruption of EGFP expression by Cas9 with sgRNAs with different initial nucleotides

608

609

610

611

612

613

614

615

616 


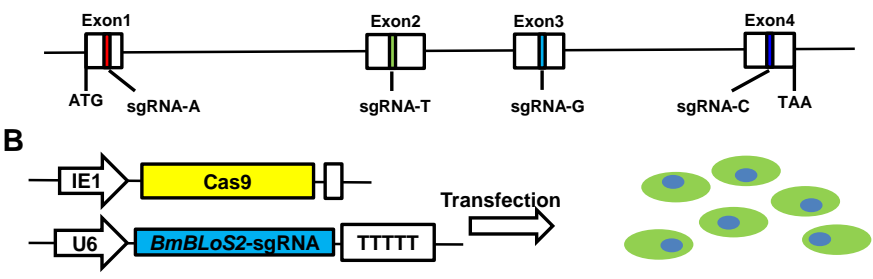

C
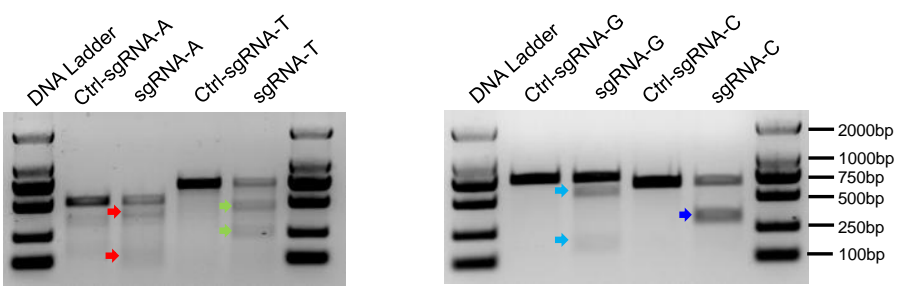

D

ATGAGTCTCAGCACAGAAGACATAGAAAAATGGACGAGAGACGGAAGAAACAAAGGTAGG ATGAGTCTCAGCACAGAAGACATAGAAAAATGGAC---AGACGGAAGAAACAAAGGTAGG ATGGGTCTCAGCACAGAAGACACAGAAAA--------AGACGGAAGAAACAAAGGTAGG ATGAGTCTCAGCACAGAAGACAT------------AGAGACGGAAGAAACAAAGGTAGG

CAGCTCTTTAAAAAAACAACTGACTACCTTCAGGCTGAAATGGTTGCTGGACAGGTGAGG CAGCTCTTTAAAAAAACAACTGACTACCTTCAGG---AAATGGTTGCTGGACAGGTGAGG T-WT CAGCTCTTTAAAAAAACAACTGACTACCTT-------AAATGGTTGCTGGACAGGTGAGG T-M1 $(-3)$ $\mathrm{T}-\mathrm{M} 2(-7)$

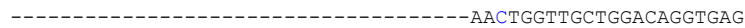
T-M3 (-39)

GATCAACAGACTCGCGATCACCAAGTACGCAGATCTAAGGAGCCTAGCCGCGAACCTGA GATCAACAGACTCGCGATCACCAAGTA------TCTAAGGAGCCTAGCCGCGAACCTGA G-WT (1) G-M2 $(-10)$ CTATAGAAATGATCCTATGAAGTAAGACGCAAATCTAAGGAGCCTAGCCGCGAACCTGAA G-M3 $(+68)$

TICGAAGTTAACGCTTACCGCCTCGACAGCTATACGAAGCAATTGAAGGCTCGTTTTAA TTCGAAGTTAACGCTTACCGCCTCGACAGCTATAC------TTGAAGGCTCGTTTTAAG

C-WT

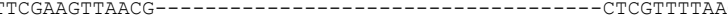

C-M1 (-7)

C-M2 $(-36)$ 

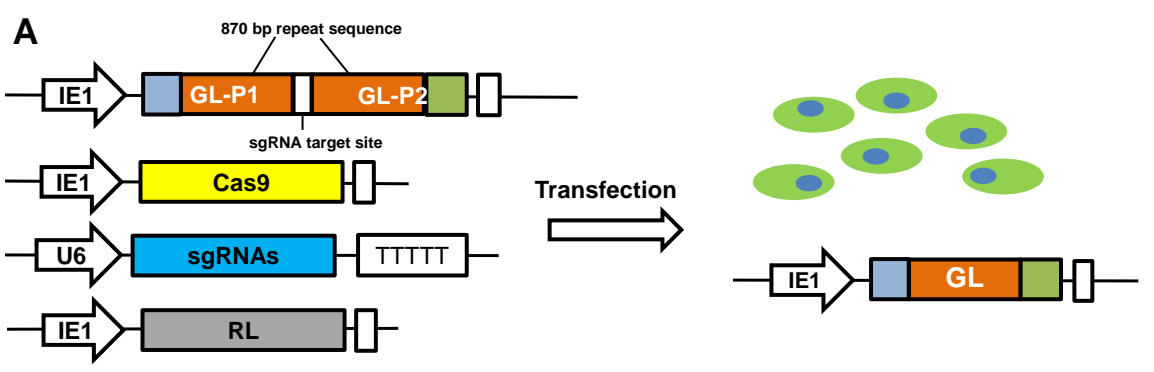

B

C

A: AgCtGAaAtGgtTGCtGGacAgG

T: TGCTGAAATGGTTGCTGGACAGG

G: GGCtGAAATGGTtGCtGGACAgG

C: CGCTGAAATGGTTGCTGGACAGG

AT rich: GGATATATATATATATATATAGG

GC rich: GGCGCGCGCGCGCGCGCGCGCGG

Control: GGCTGAAATGGTTGCTGGACATT

634

635

636

637

638

639

640

641

642

643

644

645

646

647

648

649

650

651

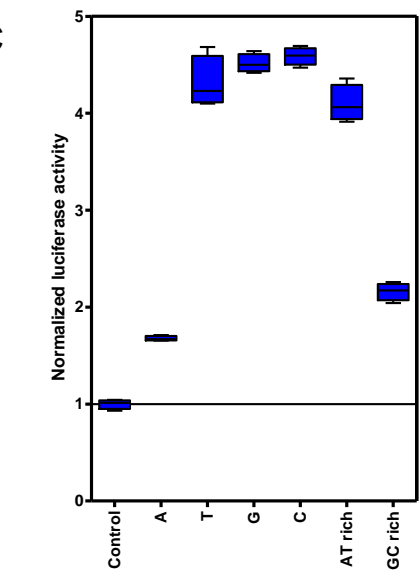

Fig. 4. Evaluation of the cleavage efficiency of N20NGG sgRNAs with a single strand-annealing (SSA) assay 


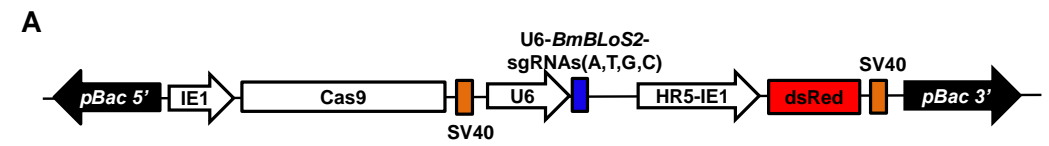

B

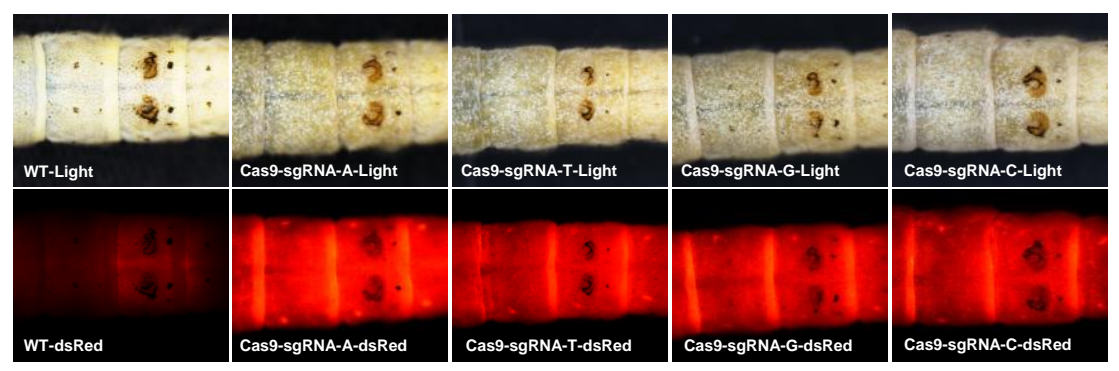

C

ATGAGTCTCAGCACAGAAGACATAGAAAAATGGACGAGAGACGGAAGAAACAAAGGTAGG ATGAGTCTCAGCACAGAAGACATAGAAAAATGGA----AGACGGAAGAAACAAAGGTAGG ATGAGTCTCAGCACAGAAGACATAGAAAA-------AGAGACGGAAGAAACAAAGGTAGG

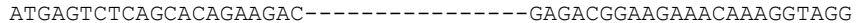

A-WT

$\mathrm{A}-\mathrm{M} 1(-4)$

A-M2 (-7)

A-M3 $(-16)$

CAGCTCTTTAAAAAAACAACTGACTACCTTCAGGCTGAAATGGTTGCTGGACAGGTGAGG CAGCTCTTTAAAAAAACAACTGACTACCTTC------AAATGGTTGCTGGACAGGTGAGG

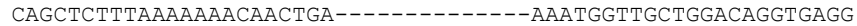

T-WT $\mathrm{T}-\mathrm{M} 1(-6)$

T-M2 (-14)

$\mathrm{T}-\mathrm{M} 3(-66)$

GATCAACAGACTCGCGATCACCAAGTACGCAGATCTAAGGAGCCTAGCCGCGAACCTGAA GATCAACAGACTCGCGATCACCAAGT----------AGGAGCCTAGCCGCGAACCTGAA GATCAACAGACTCGCGATCACCAAGT--.--_-_-----AGCCTAGCCGCGAACCTGAA GATCAACAGACTCGCGATCACCAAGT---------------CTAGCCGCGAACCTGAA

TTCGAAGTTAACGCTTACCGCCTCGACAGCTATACGAAGCAATTGAAGGCTCGTTTTAAG TTCGAAGTTAACGCTTACCGCCTCGACAGC------------TGAAGGCTCGTTTTAAG

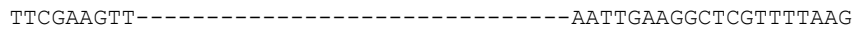

\section{G-WT}

G-M1 (-11)

G-M2 (-14)

G-M3 (-17)

C-WT

C-M2 (-13)

C-M1 (-31)

C-M3 (-91)

656 Fig. 5. Silkworms with oily skin phenotype obtained by transgenic expression of BmBLoS2 

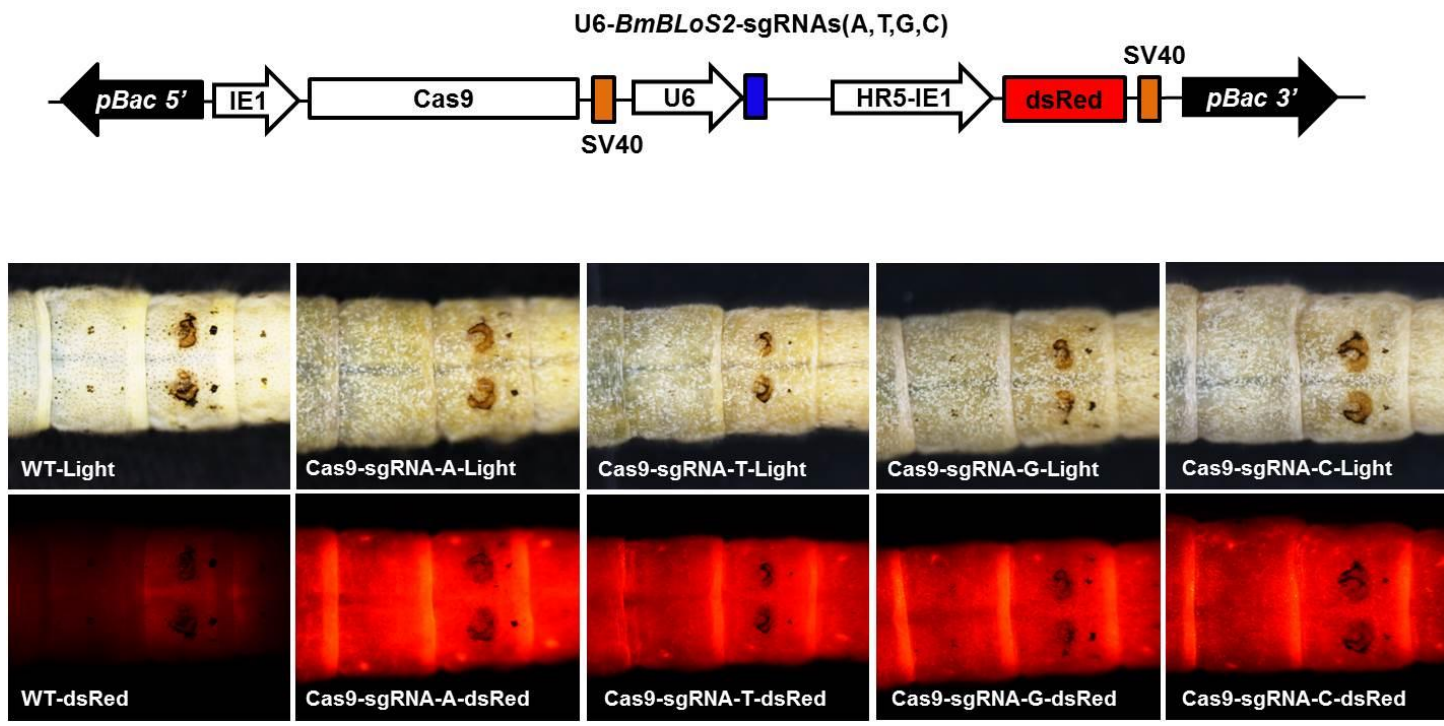